\title{
Intensified Beclin-1 Mediated by Low Expression of Mir-30a-5p Promotes Chemoresistance in Human Small Cell Lung Cancer
}

\author{
Xiang Yang ${ }^{a}$ Fan Bai ${ }^{a} \quad$ Yichen Xu ${ }^{b} \quad$ Yitian Chen ${ }^{a, b} \quad$ Longbang Chen ${ }^{a, b}$ \\ aDepartment of Medical Oncology, Jinling Hospital, Second Military Medical University, Nanjing, \\ 'Department of Medical Oncology, Jinling Hospital, School of Medicine, Nanjing University, Nanjing, \\ China
}

\section{Key Words}

Beclin-1 $\cdot$ MiR-30a-5p • Small cell lung cancer $・$ Chemoresistance $•$ Autophagy

\begin{abstract}
Background/Aims: Although small cell lung cancer $(\mathrm{SCLC})$ is sensitive to initial chemotherapy, patients experience tumor recurrence and metastasis, leading to treatment failure. Autophagy as a protective pattern for cell survival in the harsh environment plays an important role in chemoresistance. However, the role of Beclin-1, a key regulator of autophagy in the drugresistance of SCLC cells is still poorly understood. In the current study, we focused on the effect and regulation of Beclin-1 in chemoresistance of SCLC cells. Methods: We analyzed the levels of Beclin-1 in etoposide/cisplatin (EP) -resistant and -sensitive cell lines, as well as the relationship between Beclin-1 and patients' chemosensitivity. The function of Beclin-1 in chemoresistant SCLC cells in vitro was measured by MTT, WB, colony formation and flow cytometric analysis. Further rescue experiment was performed after co-transfected with siBeclin-1 and miR-30a mimics or inhibitor. Results: Beclin-1 was upregulated in drug-resistant cells and patients with lower sensitivity to etoposide/cisplatin therapy. Downregulated Beclin-1 attenuated drug sensitivity and colony formation ability of chemoresistant cells. Moreover, inhibition of Beclin-1 resulted in a dramatic decline of autophagy and increase of apoptosis in drug-resistant cells, accompanied by a remarkable reduction in $\mathrm{S}$ phase and a raise in G2/M phase of cell cycle. The transfection with miR-30a-5p mimics exhibited an opposite effect. In addition, inhibition of Beclin-1 could partly reverse the effect induced by miR-30a$5 p$ suppression in drug-sensitive cells. Conclusion: Beclin-1 regulated by miR-30a-5p plays a notable role in the drug-resistance of SCLC. Inhibition of Beclin-1 by induction of miR$30 a-5 p$ may improve the therapeutic outcome via resensitizing the drug-resistant cells to chemotherapy in SCLC.

Yitian Chen

and Longbang Chen

Department of Medical Oncology, Jinling Hospital, Second Military Medical University, 305 East Zhongshan Road, Nanjing, (China)

Tel. +862580860053, E-Mail yitianchen@126.com, drchenlb@126.com
\end{abstract}




\section{Introduction}

SCLC is a special type of lung cancer with higher malignancy, more aggressive, and earlier occurrence of metastasis [1]. The combination of etoposide (VP16) combined with cisplatin (DDP) is the first-line chemotherapy for SCLC, which often has to confront drug resistance [2]. Furthermore, the effect of secondary chemotherapy is unsatisfactory, as no effective standard treatment strategy for recurrent SCLC has been established [3]. Although there were some clinical studies on the use of targeted receptor tyrosine kinase (RTK) in the treatment of recurrent SCLC, the disclosed results were far less than being expected $[4,5]$. The reason for the failure may be that the proliferation of SCLC is not dependent on these RTKs, and the pathogenesis and driving genes of SCLC are still uncertain [6]. Therefore, the generation of resistance to chemotherapy is the primary obstacle to the clinical efficacy of SCLC patients.

Mechanism of chemoresistance includes diversity factors including: regulation of multidrug resistance protein family, cell apoptosis pathway, autophagy, intracellular drug metabolizing enzyme system, tumor stem cells, miRNA regulation network, homeostasis changes, DNA damage repair and other epigenetic regulations [7]. Autophagy, which is particularly activated in condition of metabolic stress, is a process of degradation and homeostatic cellular recycling of unnecessary or dysfunctional cytoplasmic components for energy utilization in all living cells [8,9]. Dysregulation of autophagy is common among a wide range of cancers and presents to be a novel mechanism for enhancing current anticancer treatments and overcoming chemotherapy resistance $[10,11]$. Therefore, the recent studies have pay special attention to the mechanisms of autophagy underlying chemo-resistance, revealing the potential role of autophagy as a novel treatment strategy for anticancer therapy [12].

Beclin-1 is the first autophagy related protein found in mammals, which plays an important role in the activation of autophagy and the regulation of multiple cellular signaling pathways of tumor [13]. Beclin-1 can combine with anti-apoptotic protein Bcl2 , thus inducing apoptosis. Besides, Beclin-1 has 3 domains which can interact with and constitute multiple regulatory pathways (Beclin-1-PI3KC3-Vpsl5 and Beclin-1-Bcl-2/Bcl$\mathrm{XL}$ ), and thereby regulates the development of tumors [14]. The expression level of Beclin-1 was found to be dysregulated and related to the clinical prognostic in divers types of cancer $[15,16]$. Moreover, Beclin-1 was upregulated after cisplatin treatment, resulting in enhanced activity of autophagy and drug-resistance to treatment [17]. The relationship between Beclin-1, autophagy and antitumor drugs is very complicated, remaining to be clarified.

MicroRNA is a class of noncoding RNA with about 20-25 nucleotides in length, which is a negative regulator of target genes at the post transcriptional level and is widely involved in cell growth, proliferation, differentiation and apoptosis $[18,19]$. More and more abnormal expressed miRNAs in different tumors have been reported. Consequently, miRNA involved in many aspects of tumorigenesis including proliferation, invasion and metastasis, is considered to play versatile role of oncogenes or tumor suppressor genes [20-22]. Therefore, miRNA can be used as a potential and effective target for the diagnosis and treatment of a variety of tumors. In addition, the role of miRNA in the diagnosis and treatment of cancer provides a new strategy in the field of cancer therapy [23, 24].

Our previous studies have shown that drug-resistant SCLC cell lines Letp/EP and H446/ EP have multidrug resistance. Compared with their parental cell lines, VP16/DDP-resistant SCLC cell H446/EP exhibited an increased autophagic activity, which was partly regulated by miR-24/ATG4A axis and played a pro-survival role to deteriorate therapeutic outcomes [25]. However, the expression status and therapeutic clinical roles of Beclin-1-dependent autophagy remain to be defined. This study aimed to identify the Beclin-1 involved in the formation of SCLC resistance phenotype and expression and clinical significance of it. Furthermore, overexpressed miR-30a-5p resulted in downregulated Beclin-1 and reinforced efficiency of VP16/DDP chemotherapy. The study is the first to confirm that miR-30a-5p/ 


\section{Cellular Physiology Cell Physiol Biochem 2017;43:1126-1139 \begin{tabular}{l|l|l} 
DOI: 10.1159/000481754 & $\begin{array}{l}\text { O 2017 The Author(s). Published by S. Karger AG, Basel } \\
\text { www.karger.com/cpb }\end{array}$ \\
\hline
\end{tabular} \\ Yang et al.: Mir-30a-5p/Beclin-1 Signaling Axis Plays a Role in Chemoresistance in Human Small Cell Lung Cancer}

Beclin-1 signaling axis plays a role in the mechanism of chemoresistant phenotype in SCLC, which could turn into a potential therapeutic target.

\section{Materials and Methods}

\section{Patient tissue specimens}

Tissues were collected from patients who underwent SCLC prior to chemotherapy and were histologically and clinically diagnosed via operation or biopsy between January 2001 and January 2011 at the Department of Medical Oncology, Jinling Hospital, Second Military Medical University (Nanjing, China). Tumor staging was performed using tumor-node-metastasis (TNM) classification according to the seventh edition Union for International Cancer Control (UICC) cancer staging systems. All patients were primarily treated with 4-8 courses of EP regimen chemotherapy (VP-6 $100 \mathrm{mg} / \mathrm{m}^{2}$ with DDP $60 \mathrm{mg} / \mathrm{m}^{2}$ ). This study was approved by the Ethics Committee of Jinling Hospital of Nanjing University. The samples were collected after receiving informed consent from the patients.

\section{Immunohistochemistry}

Paraffin-embedded SCLC specimens were cut into $4-\mu \mathrm{m}$ sections and deparaffinized. The sections were incubated at $4^{\circ} \mathrm{C}$ with anti-Beclin-1 rabbit antibody (ab62557, 1:100; Abcam, Inc., Cambridge, UK) overnight for staining. The secondary antibody (Dako Real Envision HRP K5007; Dako Denmark A/S, Glostrup, Denmark) was employed for 30 minutes at room temperature according to the protocol. The sections were independently evaluated and scored by two pathologists who were blinded to the patients' clinical data. Discordant cases were appraised by a consultation and a consensus was reached. The intensity of cell staining was scored as either 0 , unstained; 1 , weakly stained; 2, medium intensity; or 3, strongly stained. The proportion of cells expressing Beclin-1 was scored as follows: 0 (no expression), 1 (0-25\%), $2(26-50 \%)$, or $3(>50 \%)$. The intensity score and proportion score were multiplied to calculate the total score, which was then divided into no/low expression $(\leq 6)$ and high expression $(>6)$.

\section{Cell lines and cell culture}

Human small cell lung cancer H446 and Letp cells were obtained from the Tumor Cell Bank of the Chinese Academy of Medical Science (Shanghai, China) and cultured in RPMI 1640 medium containing 10\% fetal bovine serum and ampicillin and streptomycin at $37^{\circ} \mathrm{C}$ in a humidified atmosphere of $5 \% \mathrm{CO}_{2}$. The VP16/DDP resistant cells (H446/EP and Letp/EP) were established and preserved in a final concentration of $1 \mu \mathrm{g} / \mathrm{ml} \mathrm{VP16}$ and $0.8 \mu \mathrm{g} / \mathrm{ml}$ DDP.

\section{SiRNA and transfection}

Beclin-1 and control siRNAs were purchased from Keygentec. miR-30a-5p inhibitor (AmiR-30a5p) and miR-30a-5p mimics (PmiR-30a-5p) were provided by Keygentec. Relative sequences: siBeclin-1: F:GGUCUAAGACGUCCAACAATT, R:UUGUUGGACGUCUUAGACCTT; siRNA-control: F:UUCUCCGAACGUGUC ACGUTT, R:ACGUGACACGUUCGGAGAATT; PmiR-30a-5p: F:UGUAAACAU CCUCGACUGGAAG, R:CUUCCAGUCGAGGAUGUUUACA; AmiR-30a-5p: CU UCCAGUCGAGGAUGUUUACA. Cells were transfected with siRNAs using Lipofectamine 3000 (Invitrogen, USA), according to the manufacturer's protocol. The cells were analyzed for 48 hours after transfection according to the manufacturer's instruction. Following transfection, cells were collected at $48 \mathrm{~h}$ for further experiments.

\section{$q R T-P C R$}

Total RNAs were extracted from the cultured cells with Trizol (Invitrogen) Reagent following the manufacturer's protocol. EZ geneTM Gel/PCR Ex Kit (Biomiga, USA) was used to detect mRNA. Quantitative PCR amplifications were performed on ROCHE LightCycler480II, in $20 \mu \mathrm{l}$ volumes containing $10 \mu \mathrm{l}$ of $2 \times$ SYBR Green PCR MasterMix. The thermal profile for real-time PCR was: $95^{\circ} \mathrm{C}$ for $5 \mathrm{~min}$; followed by 40 cycles of $95^{\circ} \mathrm{C}$ for 15 seconds, $60^{\circ} \mathrm{C}$ for 20 seconds and $72^{\circ} \mathrm{C}$ for 40 seconds. Data were normalized to GAPDH and were analyzed using the comparative ${ }^{2-\Delta \Delta} \mathrm{Ct}$ method for quantification. 


\section{Cellular Physiology Cell Physiol Biochem 2017;43:1126-1139 \begin{tabular}{l|l} 
DOI: 10.1159/000481754 & $\begin{array}{l}\text { O 2017 The Author(s). Published by S. Karger AG, Basel } \\
\text { www.karger.com/cpb }\end{array}$
\end{tabular} \\ Yang et al:: Mir-30a-5p/Beclin-1 Signaling Axis Plays a Role in Chemoresistance in Human Small Cell Lung Cancer}

\section{Western blot analysis}

Protein was extracted from the cultured cells with RIPA lysis buffer on ice. Protein concentration was determined using a Braford protein assay kit (Keygentec, China), and the proteins were separated on SDS-PAGE, and then transferred to a PVDF membrane. The membrane blots were immersed in a primary antibody of anti-Beclin-1 rabbit antibody (ab62557, 1:100; Abcam, Inc., Cambridge, UK). After incubation with horseradish hydrogen peroxide-conjugated second antibody (Dako Real Envision HRP K5007; Dako Denmark A/S, Glostrup, Denmark), G:BOX chemiXR5 system was applied to visualize the protein antigen.

\section{MTT assay}

The cell growth and viability of the SCLC cell lines were assessed by using a MTT assay. Approximately 5, 000 cells/well were seeded in a 96-well plate with different drug concentrations. The MTT assay was performed on days 1-3. After incubation, $20 \mu \mathrm{l}$ of $5 \mathrm{mg} / \mathrm{ml}$ MTT was added to each well, followed by incubation for 4 hour. After discarding the medium carefully, formazan crystals were dissolved in 150 $\mu$ l dimethyl sulfoxide. Absorbance values at $490 \mathrm{~nm}$ were normalized to the percentage survival. Each experiment was accomplished in triplicate. Dose-response curves were plotted and the half maximal inhibitory concentration (IC 50) of each chemo-drug was estimated from this standard curve.

\section{Apoptosis assay}

Cell apoptosis was evaluated by using an Annexin V/FITC-PI apoptosis detection kit (Invitrogen life technologies, USA) which quantitatively measured percentage of early apoptotic cells via flow cytometry, following the manufacturer's instructions.

\section{Colony formation assay}

Cells were exposed to referred treatments, and then seed into 6-well plates. Cells were then allowed to adhere and grow for about 10 to $14 \mathrm{~d}$, followed by fixing with methanol and staining with $0.1 \%$ crystal violet. Colonies with $\geq 50$ cells were manually counted under a dissection microscope.

\section{Luciferase Assay}

Cells were seeded into a 96-well plate and cultured for $24 \mathrm{~h}$. The pmirGLO reporter gene plasmids (pmirGLO-control, pmirGLO- Beclin1-3'UTR or pmirGLO- Beclin1-mut-3'UTR) were co-transfected with miR-NC mimic or miR-30a mimic (Keygentec, China) into the cells using Lipofectamine 3000 reagent (Invitrogen, USA). Luciferase activity was measured 48 hours after transfection using the SpectraMax M3 Luciferase Assay System (Molecular Devices, USA), following the manufacturer's instructions.

\section{Statistical analysis}

All data were presented as mean \pm SD of $\geq 3$ separate experiments. Statistical analyses were performed using SPSS 18.0 software (SPSS Inc., Chicago, IL, USA). Multiple group comparisons were analyzed with oneway ANOVA; 2-group comparisons were performed by Student's t test. The patient survival by miR-30a-5p levels were analyzed with Kaplan-Meier curves. $\mathrm{P}<0.05$ was considered to indicate a statistically significant difference.

\section{Results}

Beclin-1 was differently expressed in EP-resistant and sensitive cells

In the previous study, we established EP-resistant SCLC cell line H446/EP, which exhibited higher level of autophagy than the parental cell line H446, via continuous exposure to increasing concentration of VP16 combined with DDP [25]. To test whether the mechanism of chemo-resistance on autophagy was cell type-specific, we established another EP-resistant SCLC cell line Letp/EP through the same procedure. A MTT assay was conducted to evaluate sensitivities of both cell types to these two chemotherapy agents. The IC50 values of VP16 were $14.67 \mu \mathrm{g} / \mathrm{ml}$ and $92.31 \mu \mathrm{g} / \mathrm{ml}$ in Letp and Letp/EP, respectively; while the values of DDP were $1.53 \mu \mathrm{g} / \mathrm{ml}$ and $10.62 \mu \mathrm{g} / \mathrm{ml}$, respectively (Fig. 1A). We then estimated autophagic activity in Letp/EP. Western blot analysis showed an increase of LC3- 


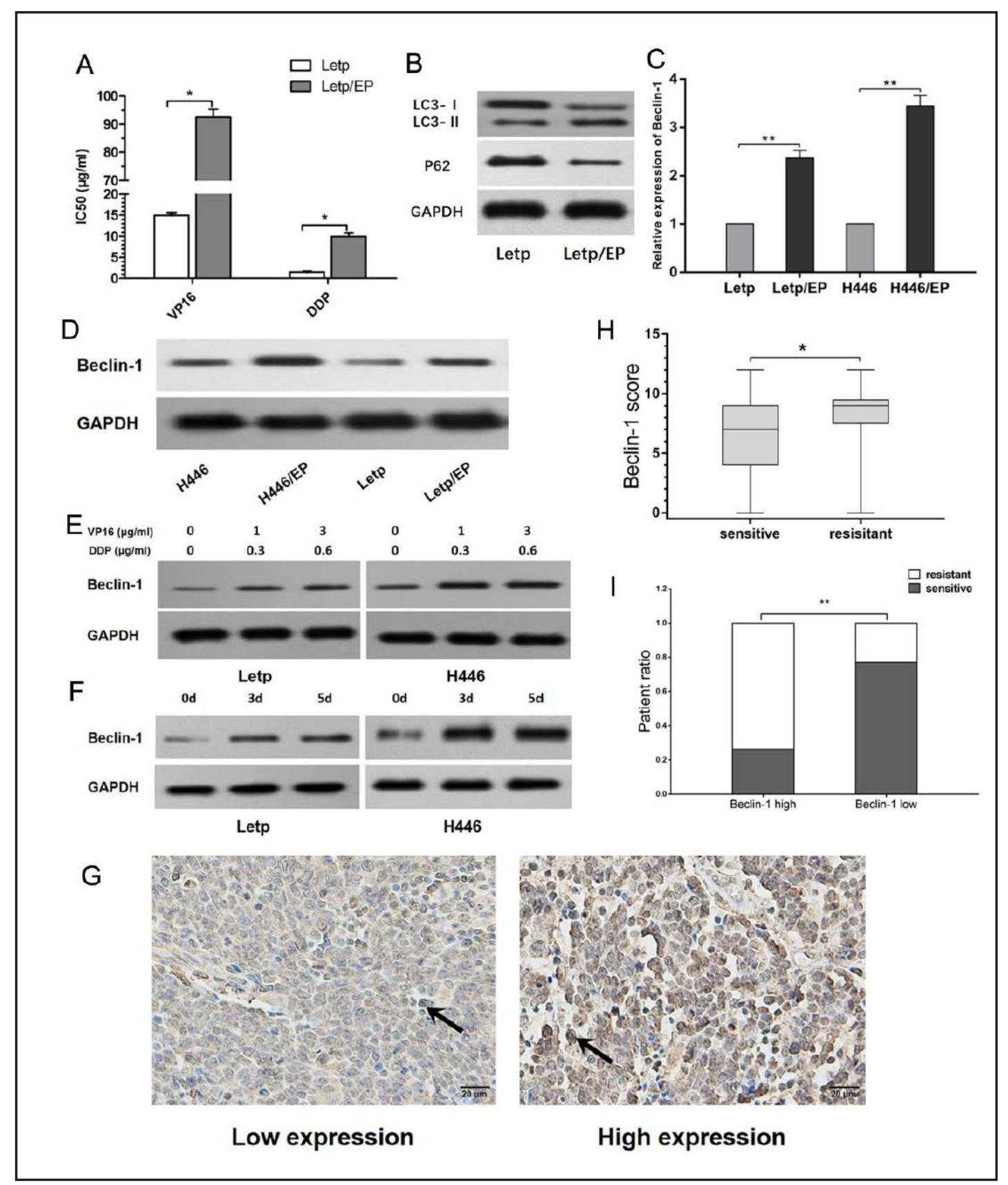

Fig. 1. Beclin-1 was upregulated in the EP-resistant SCLC cells and tissues. (A) MTT assay showed Lept/EP cells to be much more resistant to the combined VP16/DDP therapy than Letp cells. (B) Whole cell protein was analyzed by western blot using LC3 and P62 antibodies (control: GAPDH). (C, D) qRT-PCR and western blot validate Beclin-1 expression in H446/EP and Letp/EP cells via their parental cells. (E) Protein levels of Beclin-1 in SCLC cells were measured by western blot after being treated with the indicated concentrations (according to the IC50 values) of VP16 combined with DDP for $48 \mathrm{~h}$. (F) Protein levels of Beclin-1 in SCLC cells were measured by western blot after being treated with $1 \mu \mathrm{g} / \mathrm{ml}$ of VP16 and DDP for varying periods. (G) Beclin-1 protein expression was detected by immunohistochemistry, and the correlation between Beclin-1 expression and clinicopathological characteristic and prognosis was analyzed. (H) Immunohistochemical staining score of Beclin-1 in drug resistance group $(n=22)$ and drug sensitive group $(n=32)$ (I) In the patients receiving EP chemotherapy, the proportion of chemotherapy sensitivity in Beclin-1 high expression group was significantly lower than that in Beclin-1 low expression group. Error bars represent mean \pm SD from 3 independent experiments. ${ }^{*} \mathrm{P}<0.05,{ }^{*} \mathrm{P}<0.01$. 


\section{Cellular Physiology Cell Physiol Biochem 2017;43:1126-1139 \begin{tabular}{l|l|l} 
DOI: 10.1159/000481754 & $\begin{array}{l}\text { O 2017 The Author(s). Published by S. Karger AG, Basel } \\
\text { www.karger.com/cpb }\end{array}$ \\
\hline
\end{tabular} \\ Yang et al.: Mir-30a-5p/Beclin-1 Signaling Axis Plays a Role in Chemoresistance in Human Small Cell Lung Cancer}

II/LC3-I ratio and a decrease of P62 level, two selective markers of enhanced autophagy in Letp/EP cell, compared with Letp cell (Fig. 1B). To determine whether Beclin-1, a key autophagy associated protein, participated in the formation of drug-resistance in SCLC, the expression of Beclin-1 in SCLC cell lines was detected. Both of mRNA and protein expression of Beclin-1 markedly increased in H446/EP and Letp/EP cells (Fig. 1C, D). Moreover, western blot analysis showed that VP16/DDP treatment led to a dose- and timedependent increase in the expression levels of Becclin-1 (Fig. 1E, F).

Beclin-1 was upregulated in the EPresistant SCLC tissues compared with the EP-sensitive SCLC tissues

A total of 92 Chinese patients diagnosed with SCLC were retrospectively enrolled. 54 cases who received EP treatment were divided into "sensitive" (complete or partial response, $\mathrm{CR}+\mathrm{PR}$ ) and "resistant" (stable or progressive disease, SD+PD) groups based on the response to the EP regimen chemotherapy. The clinicopathological characteristics of patients were shown in Table 1. The expression of Beclin-1 was detected by immunohistochemistry and the levels of expression in tissues were evaluated via the multiplication of proportion score and intensity score (Fig. 1G). Beclin-1 was significantly upregulated in the EP-resistant group $(n=22)$ compared with the EP-sensitive group ( $n=32)$ (Fig. $1 \mathrm{H})$. When the samples were divided into two groups according to the expression level of Beclin-1, 27 cases were found to be sensitive to chemotherapy form the 35 cases of low Beclin-1 (77.14\%), while 5 cases were found to be sensitive from the 19 cases of high Beclin-1 expression (26.31\%), and the patient ratio of sensitive cases in the latter was significantly lower than the former (Fig. 1I).

Beclin-1 repression reversed the in vitro chemoresistance of SCLC cells

To determine the role of Beclin-1 involved in the SCLC cells, small interfering RNA (siRNA) against Beclin-1 of different sequences were transfected into Letp/EP and H446/ EP cells. $48 \mathrm{~h}$ after transfection, qRT-PCR confirmed the inhibitory efficiency of all sequences (Fig. 2A). Western blot was conducted to validate the inhibitory efficiency of the chosen sequence (Fig. 2B).

To further confirm the effects of Beclin-1 on regulating the chemo-sensibility of SCLC cells, siRNA-control or siBeclin-1 were transfected into Letp/EP and H446/EP cells, and the IC50 values of VP16 or DDP were measured by MTT assay. The IC50 values of VP16 (or DDP) in Letp/EP/siBeclin-1 or H446/EP/siBeclin-1 cells were significantly decreased $(\mathrm{P}<0.01)$ compared with those in control cells. (Fig. 2C).

Meanwhile, downregulation of Beclin-1 dramatically abrogated the autophagy activity of Letp/EP and H446/EP cell lines (Fig. 2D). Similarly, downregulation of Beclin-1 greatly reduced the colony formation capacities of Letp/EP and H446/EP cell lines (Fig. 2E). Moreover, flow cytometric assay was performed to assess cell apoptosis and cell cycle. The apoptosis rate of Letp/EP and Letp/EP/siRNA-control cell lines were $(4.71 \pm 0.61) \%$ and $(5.36 \pm 0.59)$ $\%$, which were raised to $(13.01 \pm 1.13) \%$ after being transfected with siBeclin-1; the apoptosis rate of H446/EP and H446/EP/siRNA-control cell lines were $(10.77 \pm 0.58) \%$ and 
$(9.68 \pm 0.68) \%$, which were raised to $(16.06 \pm 1.10) \%$ after being transfected with siBeclin-1 (Fig. 2F). Cell cycle analysis revealed that downregulation of Beclin-1 significantly increased the percentage of cells in G2/M phase in Letp/EP and H446/EP cells (Fig. 2G). Collectively, suppression of Beclin-1 could reverse the in vitro chemoresistance which was accompanied by elevated autophagic activity in SCLC cells.

Beclin-1-dependent autophagy in SCLC was directly regulated by miR-30a-5p

Having identified Beclin-1 as furtherance of SCLC progression, we became interested in identifying which miRNA might regulate Beclin-1 in SCLC. To elucidate the mechanisms by which Beclin-1 dependent autophagy was regulated, prediction of binding between miRNA

Fig. 2. Repression of Beclin-1 reversed the chemoresistance of SCLC cells in vitro. (A) qRTPCR was performed to detect the mRNA of Beclin-1 48h after transfected with different sequences of siBeclin-1 in Letp/EP and H446/EP. (B) 48h after transfection, western blot confirmed the inhibitory efficiency in Letp/EP and H446/ EP cells. (C) The IC50 values for VP16 or DDP as determined by MTT assay after transfection of siRNA-control or si-Beclin-1 into VP16/DDP-resistant SCLC cells. Downregulation of Beclin-1 reversed the chemoresistance of drug-resistant SCLC cells. (D) After transfection of siBeclin-1, the expressions of LC3 and P62 in Letp/EP and H446/EP cells were detected by western blot. (E) After transfection of siBeclin-1, the colony formation assay was conducted as described in methods and the number of colonies was counted and compared. (F) Flow cytometric analysis of apoptosis after transfection of siRNAcontrol or siBeclin-1 into Letp/ EP and H446/EP cells. (G) Flow cytometry analysis of cell cycle in Letp/EP and H446/EP cells transfected with siRNA-control or siBeclin-1. The data were the means \pm standard error of at least three independent experiments. ${ }^{*} \mathrm{P}<0.05$, ${ }^{*} \mathrm{P}<0.01$.
A
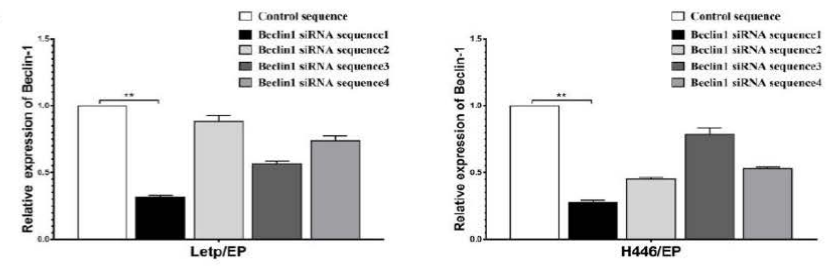

B
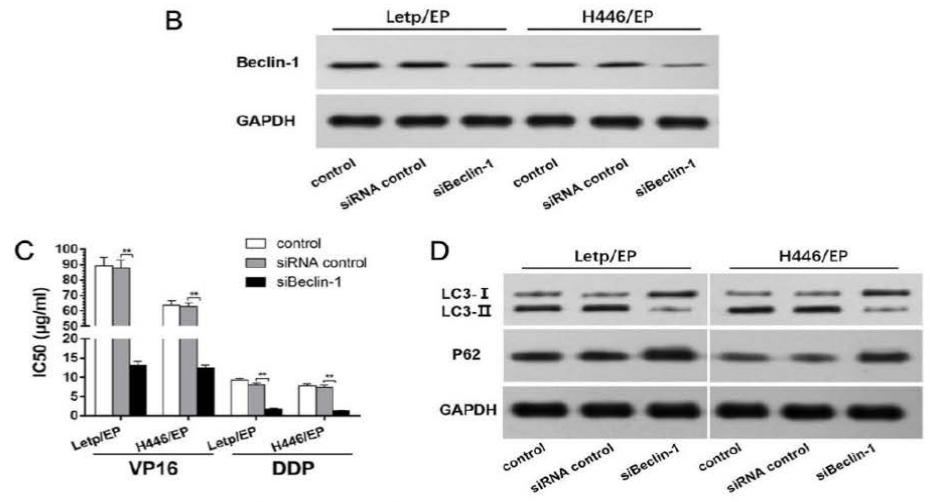

$E$
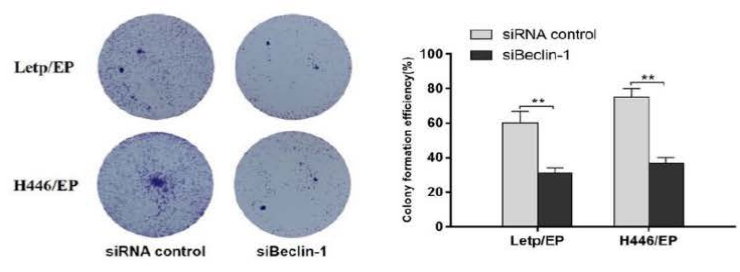

$\mathrm{F}$

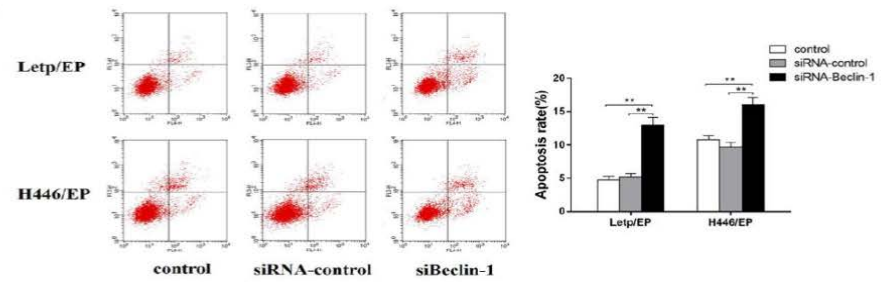

G
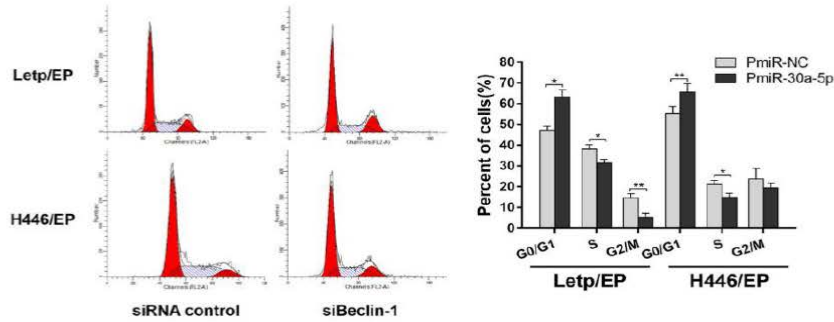
and 3'-UTR of Beclin-1 mRNA was performed using the MirDB and TargetScan bioinformatics algorithm. We found miR-30a-5p to be of special interest among other miRNAs targeting Beclin-1, because it has been recently associated with metastasis and lower survival rates in colorectal cancer [26]. Furthermore, miR-30a-5p was declared to be extraordinarily downregulated by microarray analysis in chemo-resistant cell in previous study (Fig. 3A). Consistent with the results, qRT-PCR showed a decrease of miR-30a-5p expression in Letp/EP and H446/EP cells, compared with Letp and H446 cells (Fig. 3B).

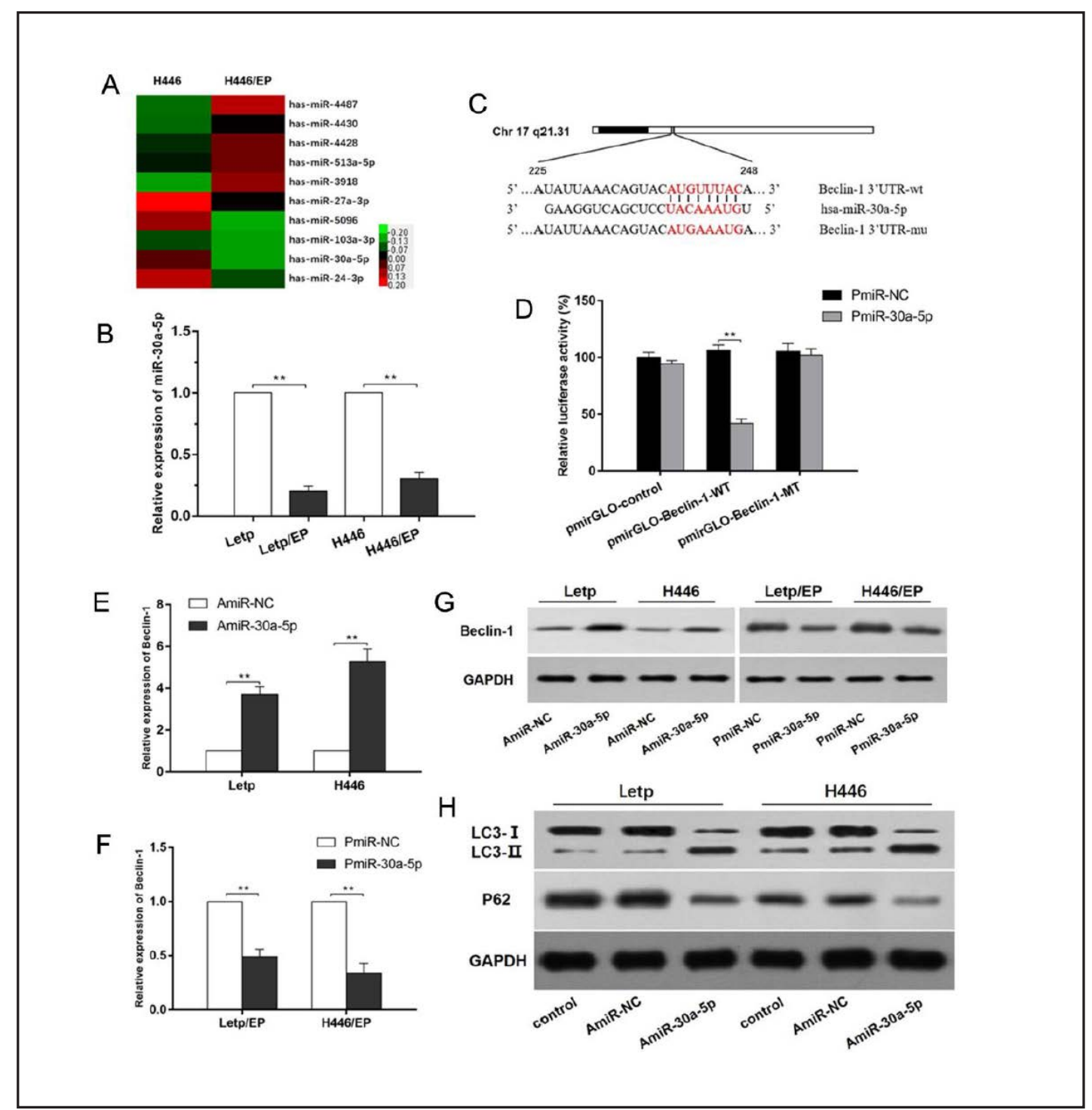

Fig. 3. Beclin-1-dependent autophagy in SCLC was directly regulated by miR-30a-5p. (A) The heat map of differently expressed 10 miRNAs between H446/EP and H446 cells, increased (red) or decreased (green) to at least 2 -fold change. Scale: log2(fold change). (B) qRT-PCR detection of relative miR-30a-5p expression in drug-resistant SCLC cells (Letp/EP and H446/EP) and their parental SCLC cells (Letp and H446). (C) The wild and mutant type of binding sequences of miR-30a-5p within human Beclin-1 3'UTR. (D) Luciferase activity analysis of Beclin-1 3'UTR (wild and mutant type) after co-transfection with PmiR-30a-5p or PmiRNC in HEK-293 cells. qRT-PCR (E, F) and Western blot (G) showed Beclin-1 expression in drug-sensitive cells transfected with AmiR-NC or AmiR-30a-5p and drug-resistant cells transfected with PmiR-NC or PmiR-30a5p. (H) The expression of LC3 and P62 were detected by Western blot to detect the activity of autophagy after being transfected with AmiR-NC or AmiR-30a-5p in Letp and H446 cells. The data were the means \pm standard error of at least three independent experiments. ${ }^{*} \mathrm{P}<0.05,{ }^{* *} \mathrm{P}<0.01$. 
To determine whether Beclin-1 was regulated by miR30a-5p through direct binding to the 3'UTR region of Beclin-1, the Beclin-1-3'UTR was inserted into a luciferase reporter vector. Transfection of miR30a-5p mimics (PmiR-30a-5p) or miRNA mimics control (PmiRNC), along with wild-type Beclin-1-3'UTR, into HEK293, Letp/EP and H446/EP cells led to a great decrease in luciferase activity compared with the co-transfection of mutant Beclin-13'UTR (Fig. 3C, D). We certificated Beclin-1 levels in SCLC cells after regulating miR-30a-5p expression. Overexpression of miR-30a-5p in H446/EP and Letp/EP cells using PmiR-30a$5 p$ decreased Beclin-1 expression, while miR-30a inhibitor (AmiR-30a) showed the opposite effect on Beclin-1 expression in H446 and Letp cells (Fig. 3E, F, and G).

In order to verify the effect of miR-30a-5p on autophagy in SCLC cells, AmiR-30a-5p was transfected into Letp and H446 cells. Western blot analysis of LC3 and P62 showed a notable enhanced autophagic activity along with the downregulation of miR-30a-5p induced by AmiR-30a-5p in SCLC cells (Fig. 3H).

MiR-30a-5p contributed to chemoresistance of SCLC cells partially in an Beclin-1dependent manner

To determine the effects of miR-30a-5p in modulating the chemoresistance in SCLC cells, we inhibited upregulated miR-30a-5p by transfecting AmiRNA in Letp and H446 cells or overexpressed downregulated miR30a-5p by transfecting PmiRNA in Letp/EP and H446/ EP cells, whose expression patterns were verified by qRT-PCR (Fig.4 A, B), and then drug sensibility was tested by MTT assay. The IC50 values of VP16 or DDP were measured by MTT assay. The IC50 values of VP16 (or DDP) in Letp/AmiR-30a-5p or H446/ AmiR-30a-5p cells significantly increased $(\mathrm{P}<0.01)$ compared with those in control cells. Nevertheless, the resistant effects could be partially suppressed by siBeclin-1 (Fig. 4C). However, cotransfection with PmiR-30a-5p and siBeclin-1, the IC50 values of Letp/EP and H446/EP cells for VP16/DDP were not significantly different from those of transfected with PmiR-30a-5p or siBeclin-1 separately (Fig. 4D).

To further verify that miR-30a-5p regulates autophagy by directly repressing Beclin-1 in SCLC cells, we performed rescue experiments through Beclin-1 downexpression. Autophagy induced by AmiR-30a-5p was repressed after co-transfection with siBeclin-1 in Letp and H446 cells; while downregulated Beclin-1 could not further attenuate autophagy inhibited by PmiR-30a in Letp/EP and H446/EP cells (Fig. 4E, F). Similarly, overexpression of Beclin-1 evidently reduced the colony formation capacities of Letp/EP and H446/EP cells (Fig. 4G).

Furthermore, flow cytometric assay was conducted to elucidate cell apoptosis and cell cycle. The apoptosis rates of Letp and H446 cells co-transfected with AmiR-30a-5p and siBeclin- 1 were $(9.78 \pm 0.48) \%$ and $(8.09 \pm 0.85) \%$, which were partly reversed compared with that of transfected with AmiR-30a-5p; Nevertheless, co-transfected with siBeclin-1 could not boost cell apoptosis induced by PmiR-30a-5p (Fig. 4H, I). Cell cycle analysis indicated that upregulation of miR-30a significantly increased the percentage of cells in G0/ G1 phase in Letp/EP and H446/EP cells (Fig. 4J). Therefore, miR-30a-5p contributed to in vitro chemoresistance of SCLC cells, at least partially in a Beclin-1-dependent manner.

\section{Discussion}

Lung cancer is one of the most common malignant tumors with the highest mortality in the world [27]. In recent years, the clinical treatment strategy of lung cancer, especially NSCLC, has been greatly improved and enriched with the development of driving genes and relevant targeted therapies. However, standardized cytotoxic anticancer drug therapy is still the cornerstone of the tumor treatment strategy, in both NSCLC and SCLC [28]. In the therapeutic process of EGFR-TKI treatment, 3-15\% of NSCLC cases appear to transform histopathologically to SCLC [29]. Recurrence of the tumor will often be resistant to drugs with different molecular structure and anti-cancer mechanism. That is called multidrug resistance (MDR), a kind of self-protection of tumor cells against a variety of antitumor drugs, leading 
A

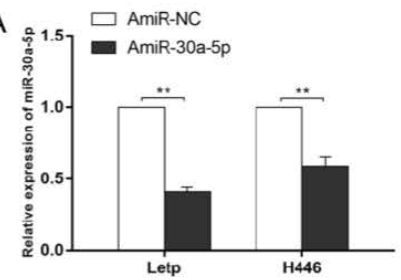

C

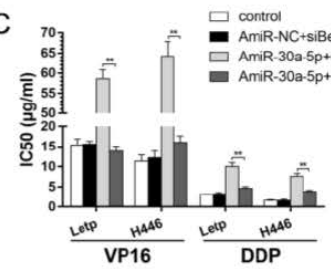

D

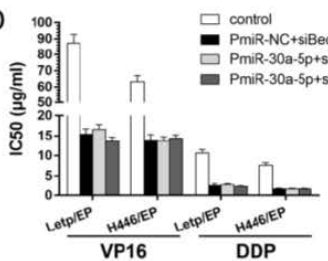

G

Letp/EP

$\mathrm{H}$

PmiR-NC

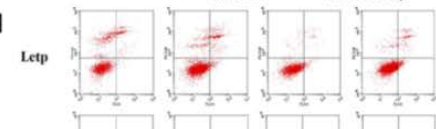

н446
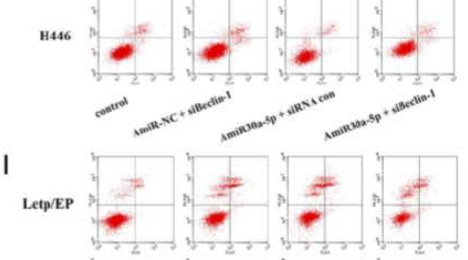

H4A6EP

H446/EP

J

Letp/EP$$
\text { (1) }
$$

mesco
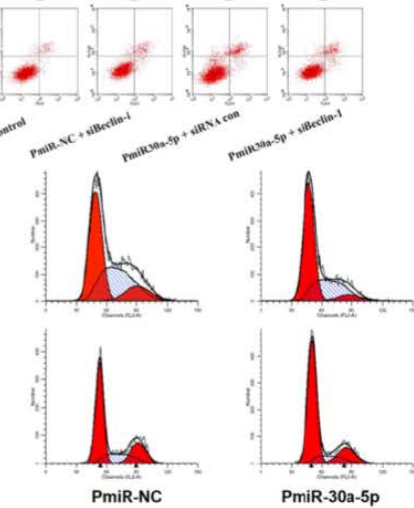

E

$\mathrm{F}$
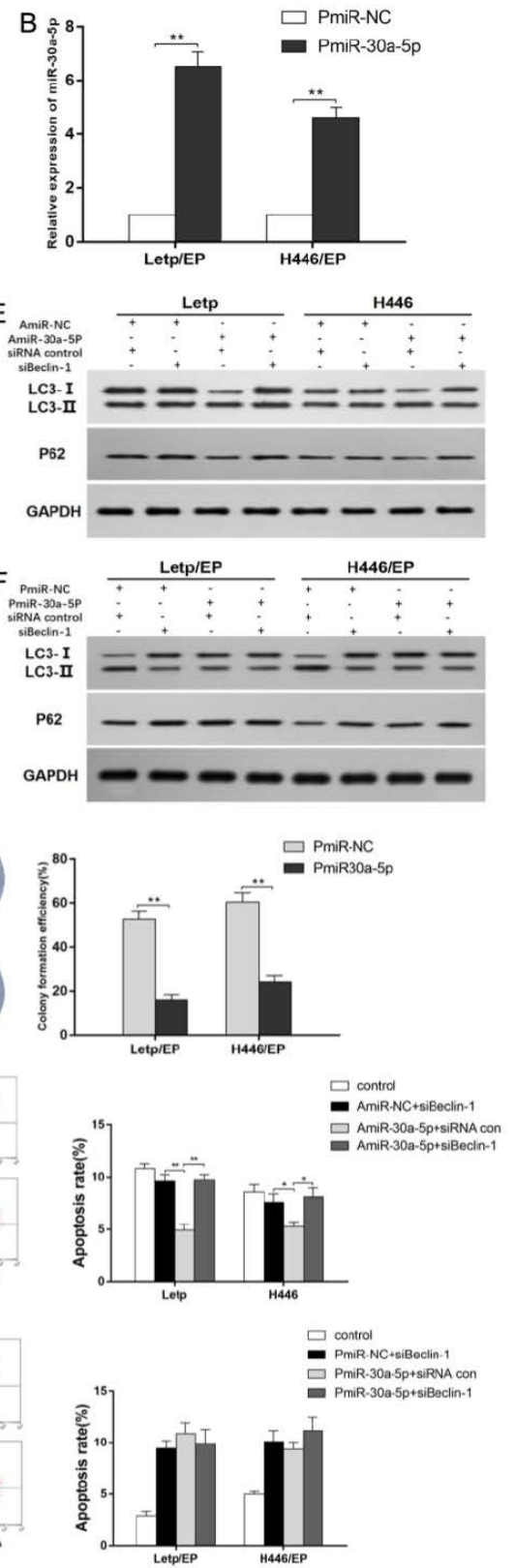

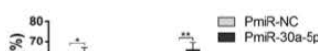

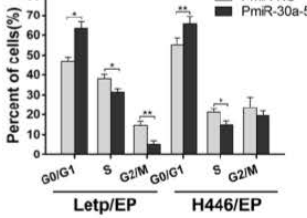

Fig. 4. MiR-30a-5p promoted chemoresistance of SCLC cells through regulating Beclin-1. (A, B) The expression of miR-30a-5p was detected by real-time quantitative PCR and the transfection efficiency was verified, after transfected with AmiR-30a-5p or AmiR-NC in Letp and H446 cells and PmiR-30a-5p or PmiR-NC in Letp/EP and H446/EP cells. (C) The IC50 values of VP16 or DDP determined by MTT assay after being trans- 
fected with AmiR-NC or AmiR-30a-5p into Letp and H446 cells with co-transfection with siRNA-control or siBeclin-1. (D) The IC50 values of VP16 or DDP determined by MTT assay after being transfected with PmiRNC or PmiR-30a-5p into Letp/EP and H446/EP cells with co-transfection with siRNA-control or siBeclin-1. (E) Protein levels of LC3 and P62 were measured by western blot after transfection of siRNA-control or siBeclin-1 into Letp and H446 cells, with co-transfection of AmiR-NC or AmiR-30a-5p. (F) Protein levels of LC3 and P62 were measured by western blot after transfection of siRNA-control or siBeclin-1 into Letp/EP and H446/EP cells, with co-transfection of PmiR-NC or PmiR-30a-5p. (G) The colony formation assay was executed as described in methods. The number of colonies was counted and compared. (H) Flow cytometric analysis of apoptosis after co-transfection with siBeclin-1 and AmiR-30a-5p into Letp and H446 cells. (I) Flow cytometric analysis of apoptosis after co-transfection with siBeclin-1 and PmiR-30a-5p into Letp/EP and H446/EP cells. (J) Flow cytometry analysis of cell cycle in Letp/EP and H446/EP cells. The data were the means \pm standard error of at least three independent experiments. ${ }^{*} \mathrm{P}<0.05,{ }^{* *} \mathrm{P}<0.01$.

to more than $90 \%$ failure in the treatment [30]. The mechanism underlying the formation of chemotherapy resistance phenotype remains unclear, for the reason of individual differences in the tumor patients in the aspects of clinical manifestation, prognosis, treatment response and tolerance, as well as the interaction among the various types of chemotherapy drugs. In our previous study, VP16/DDP-resistant cell line H446/EP exhibited obvious abnormality of autophagy associated with tumor chemoresistance in SCLC.

Beclin-1, as the core molecules in autophagy regulation by forming complexes with multiple proteins, was originally identified as an interacting protein with Bcl-2 [31-33]. The inhibition of apoptosis by Beclin-1 may exert through the interaction with Bcl-2, and the interaction with Beclin-1 did not make Bcl-2 lose its antiapoptotic potential [34]. Beclin-1 gene heterozygous mice showed severe autophagy defects and tumor susceptible phenotype [35]. Knocking down Beclin-1 or using chloroquine, could further promote the apoptosis induced by the JAK2/STAT3 inhibitor [36]. Further research demonstrated that downregulation of Beclin-1 and upregulation of Bcl-XL were associated with high degree of malignancy and poor prognosis in hepatocellular carcinoma [37]. Therefore, Beclin-1 played a decisive role in autophagy and apoptosis, and could be conducted as a target for cancer therapy [38]. In this study, Beclin-1 was evidently overexpressed in drug-resistant cells, consistent with in clinical cases. Besides, Beclin-1 was gradually elevated along with the concentration increased or time extension of anticancer drugs. Further downregulating Beclin-1 resensitized drug-resistant cells to chemotherapy via inhibiting autophagy and inducing apoptosis.

In individualized treatment, miRNAs could be used to predict the invasion and the response to anticancer drug therapy $[39,40]$. Using bioinformatics methods combining with miRNA array, we screened out miR30a-5p, the pivotal miRNA regulating the expression of Beclin-1, for further study on its effect in drug-resistance of SCLC cells. As a member of miRNA, miR-30a-5p has been reported to inhibit the cell proliferation, invasion, migration and autophagy in some types of tumors $[41,42]$. However, miR-30a-5p acted as oncogene because of different target genes in other tumors [43]. Furthermore, the expression level of miR-30a-5p in chemoresistant ovarian cancer cells was significantly higher than that in the sensitive cells, and was involved in the formation of drug-resistant phenotype [44]. Although miR-30a-5p has been verified to play a regulatory role in the development and progression of a variety of tumors, its role in the formation of drug-resistant phenotype in SCLC is unclear. We clarified that inhibition of autophagy by induced overexpression of miR-30a- $5 p$ helps to resensitize SCLC cells to VP16/DDP therapy.

Altogether, the expression of Beclin-1 in SCLC and its relationship with clinicopathological features and chemotherapeutic response were analyzed, and the role of abnormal regulation of miR-30a-5p/Beclin-1 signaling axis in the chemotherapy resistance of SCLC cells was investigated. It would provide a novel data and strategies for reversing the drug resistance and clinical individualized treatment of SCLC. 
Yang et al:: Mir-30a-5p/Beclin-1 Signaling Axis Plays a Role in Chemoresistance in Human Small Cell Lung Cancer

However, resistance to chemotherapy is a complicated dynamic phenomenon and the processes of apoptosis and autophagy are transformative under different conditions and in various periods, hence the exact mechanism of cell death remains to be elucidated. Whether miR-30a-5p has other downstream target genes involved in the formation of chemoresistance in SCLC is uncertain. Further research is needed to provide theoretical basis for exploring effective biomarkers and therapeutic targets for personalized therapy.

\section{Acknowledgements}

This work was supported by the National Natural Science Foundation of China [grant number 81402492].

\section{Disclosure Statement}

The authors have no conflict of interest.

\section{References}

1 Lopez-Chavez A, Sandler A: Systemic issues in small cell lung cancer. Curr Probl Cancer 2012;36:131-155.

-2 Lara PN, Jr., Chansky K, Shibata T, Fukuda H, Tamura T, Crowley J, Redman MW, Natale R, Saijo N, Gandara DR: Common arm comparative outcomes analysis of phase 3 trials of cisplatin + irinotecan versus cisplatin + etoposide in extensive stage small cell lung cancer: final patient-level results from Japan Clinical Oncology Group 9511 and Southwest Oncology Group 0124. Cancer 2010;116:5710-5715.

-3 Minami T, Kijima T, Kohmo S, Arase H, Otani Y, Nagatomo I, Takahashi R, Miyake K, Higashiguchi M, Morimura O, Ihara S, Tsujino K, Hirata H, Inoue K, Takeda Y, Kida H, Tachibana I, Kumanogoh A: Overcoming chemoresistance of small-cell lung cancer through stepwise HER2-targeted antibody-dependent cellmediated cytotoxicity and VEGF-targeted antiangiogenesis. Sci Rep 2013;3:2669.

4 Johnson BE, Fischer T, Fischer B, Dunlop D, Rischin D, Silberman S, Kowalski MO, Sayles D, Dimitrijevic S, Fletcher C, Hornick J, Salgia R, Le Chevalier T: Phase II study of imatinib in patients with small cell lung cancer. Clin Cancer Res 2003;9:5880-5887.

5 Moore AM, Einhorn LH, Estes D, Govindan R, Axelson J, Vinson J, Breen TE, Yu M, Hanna NH: Gefitinib in patients with chemo-sensitive and chemo-refractory relapsed small cell cancers: a Hoosier Oncology Group phase II trial. Lung Cancer 2006;52:93-97.

6 Fischer B, Marinov M, Arcaro A: Targeting receptor tyrosine kinase signalling in small cell lung cancer (SCLC): what have we learned so far? Cancer Treat Rev 2007;33:391-406.

7 Wu Q, Yang Z, Nie Y, Shi Y, Fan D: Multi-drug resistance in cancer chemotherapeutics: mechanisms and lab approaches. Cancer Lett 2014;347:159-166.

-8 Wu X, Feng X, Zhao X, Ma F, Liu N, Guo H, Li C, Du H, Zhang B: Role of Beclin-1-Mediated Autophagy in the Survival of Pediatric Leukemia Cells. Cell Physiol Biochem 2016;39:1827-1836.

-9 Zhou Z, You Z: Mesenchymal Stem Cells Alleviate LPS-Induced Acute Lung Injury in Mice by MiR-142a-5pControlled Pulmonary Endothelial Cell Autophagy. Cell Physiol Biochem 2016;38:258-266.

$>10$ Duffy A, Le J, Sausville E, Emadi A: Autophagy modulation: a target for cancer treatment development. Cancer Chemother Pharmacol 2015;75:439-447.

11 Li C, Liu Y, Liu H, Zhang W, Shen C, Cho K, Chen X, Peng F, Bi Y, Hou X, Yang Z, Zheng Z, Wang K, Wang X, Zhang J, Zhong C, Zou H, Zhang X, Zhao S: Impact of autophagy inhibition at different stages on cytotoxic effect of autophagy inducer in glioblastoma cells. Cell Physiol Biochem 2015;35:1303-1316.

12 O'Donovan TR, O'Sullivan GC, McKenna SL: Induction of autophagy by drug-resistant esophageal cancer cells promotes their survival and recovery following treatment with chemotherapeutics. Autophagy 2011;7:509-524.

13 Maiuri MC, Tasdemir E, Criollo A, Morselli E, Vicencio JM, Carnuccio R, Kroemer G: Control of autophagy by oncogenes and tumor suppressor genes. Cell Death Differ 2009;16:87-93. 


\section{Cellular Physiology Cell Physiol Biochem 2017;43:1126-1139 \begin{tabular}{l|l|l}
\hline DOI: 10.1159/000481754 & $\begin{array}{l}\text { C) } 2017 \text { The Author(s). Published by S. Karger AG, Basel } \\
\text { www.karger.com/cpb }\end{array}$ \\
\hline
\end{tabular}

Yang et al:: Mir-30a-5p/Beclin-1 Signaling Axis Plays a Role in Chemoresistance in Human Small Cell Lung Cancer

14 Kang R, Zeh HJ, Lotze MT, Tang D: The Beclin 1 network regulates autophagy and apoptosis. Cell Death Differ 2011;18:571-580.

15 Lin HX, Qiu HJ, Zeng F, Rao HL, Yang GF, Kung HF, Zhu XF, Zeng YX, Cai MY, Xie D: Decreased expression of Beclin 1 correlates closely with Bcl-xL expression and poor prognosis of ovarian carcinoma. PLoS One 2013;8:e60516.

16 Yu M, Gou WF, Zhao S, Xiao LJ, Mao XY, Xing YN, Takahashi H, Takano Y, Zheng HC: Beclin 1 expression is an independent prognostic factor for gastric carcinomas. Tumour Biol 2013;34:1071-1083.

-17 Zou Z, Wu L, Ding H, Wang Y, Zhang Y, Chen X, Chen X, Zhang CY, Zhang Q Zen K: MicroRNA-30a sensitizes tumor cells to cis-platinum via suppressing beclin 1-mediated autophagy. J Biol Chem 2012;287:41484156.

-18 Fan T, Wang W, Zhang B, Xu Y, Chen L, Pan S, Hu H, Geng Q: Regulatory mechanisms of microRNAs in lung cancer stem cells. Springerplus 2016;5:1762.

19 Yates LA, Norbury CJ, Gilbert RJ: The long and short of microRNA. Cell 2013;153:516-519.

20 Calin GA, Sevignani C, Dumitru CD, Hyslop T, Noch E, Yendamuri S, Shimizu M, Rattan S, Bullrich F, Negrini M, Croce CM: Human microRNA genes are frequently located at fragile sites and genomic regions involved in cancers. Proc Natl Acad Sci U S A 2004;101:2999-3004.

21 Ge YZ, Xin H, Lu TZ, Xu Z, Yu P, Zhao YC, Li MH, Zhao Y, Zhong B, Xu X, Zhou LH, Wu R, Xu LW, Wu JP, Li WC, Zhu JG, Jia RP: MicroRNA expression profiles predict clinical phenotypes and prognosis in chromophobe renal cell carcinoma. Sci Rep 2015;5:10328.

-22 Zhi Y, Pan J, Shen W, He P, Zheng J, Zhou X, Lu G, Chen Z, Zhou Z: Ginkgolide B Inhibits Human Bladder Cancer Cell Migration and Invasion Through MicroRNA-223-3p. Cell Physiol Biochem 2016;39:1787-1794.

-23 Long W, Zhao C, Ji C, Ding H, Cui Y, Guo X, Shen R, Liu J: Characterization of serum microRNAs profile of PCOS and identification of novel non-invasive biomarkers. Cell Physiol Biochem 2014;33:1304-1315.

-24 Wang QX, Zhu YQ Zhang H, Xiao J: Altered MiRNA expression in gastric cancer: a systematic review and meta-analysis. Cell Physiol Biochem 2015;35:933-944.

-25 Pan B, Chen Y, Song H, Xu Y, Wang R, Chen L: Mir-24-3p downregulation contributes to VP16-DDP resistance in small-cell lung cancer by targeting ATG4A. Oncotarget 2015;6:317-331.

-26 Liu L, Meng T, Wang QS, Jin HZ, Sun ZQ Jin B, Fang F, Wang HJ: Association of Beclin-1 and microRNA-30a expression with the severity and treatment response of colorectal cancer. Genet Mol Res 2016;15:

27 Hirsch FR, Scagliotti GV, Mulshine JL, Kwon R, Curran WJ, Jr., Wu YL, Paz-Ares L: Lung cancer: current therapies and new targeted treatments. Lancet 2017;389:299-311.

28 Kim ES: Chemotherapy Resistance in Lung Cancer. Adv Exp Med Biol 2016;893:189-209.

29 Dorantes-Heredia R, Ruiz-Morales JM, Cano-Garcia F: Histopathological transformation to small-cell lung carcinoma in non-small cell lung carcinoma tumors. Transl Lung Cancer Res 2016;5:401-412.

-30 Pluchino KM, Hall MD, Goldsborough AS, Callaghan R, Gottesman MM: Collateral sensitivity as a strategy against cancer multidrug resistance. Drug Resist Updat 2012;15:98-105.

-31 Luo S, Rubinsztein DC: Apoptosis blocks Beclin 1-dependent autophagosome synthesis: an effect rescued by Bcl-xL. Cell Death Differ 2010;17:268-277.

-32 Liang XH, Jackson S, Seaman M, Brown K, Kempkes B, Hibshoosh H, Levine B: Induction of autophagy and inhibition of tumorigenesis by beclin 1. Nature 1999;402:672-676.

33 Wang XM, Yang YJ, Wu YJ, Zhang Q, Qian HY: Attenuating Hypoxia-Induced Apoptosis and Autophagy of Mesenchymal Stem Cells: the Potential of Sitagliptin in Stem Cell-Based Therapy. Cell Physiol Biochem 2015;37:1914-1926.

-34 Ciechomska IA, Goemans GC, Skepper JN, Tolkovsky AM: Bcl-2 complexed with Beclin-1 maintains full antiapoptotic function. Oncogene 2009;28:2128-2141.

-35 Qu X, Yu J, Bhagat G, Furuya N, Hibshoosh H, Troxel A, Rosen J, Eskelinen EL, Mizushima N, Ohsumi Y, Cattoretti G, Levine B: Promotion of tumorigenesis by heterozygous disruption of the beclin 1 autophagy gene. J Clin Invest 2003;112:1809-1820.

-36 Kim SY, Song X, Zhang L, Bartlett DL, Lee YJ: Role of Bcl-xL/Beclin-1 in interplay between apoptosis and autophagy in oxaliplatin and bortezomib-induced cell death. Biochem Pharmacol 2014;88:178-188.

-37 Dalby KN, Tekedereli I, Lopez-Berestein G, Ozpolat B: Targeting the prodeath and prosurvival functions of autophagy as novel therapeutic strategies in cancer. Autophagy 2010;6:322-329. 


\section{Cellular Physiology Cell Physiol Biochem 2017;43:1126-1139}

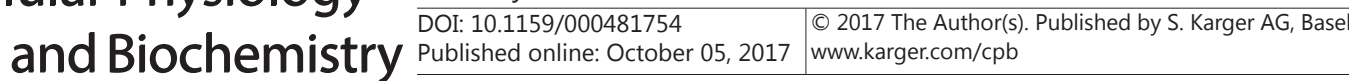

Yang et al:: Mir-30a-5p/Beclin-1 Signaling Axis Plays a Role in Chemoresistance in Human

38 Li Z, Chen B, Wu Y, Jin F, Xia Y, Liu X: Genetic and epigenetic silencing of the beclin 1 gene in sporadic breast tumors. BMC Cancer 2010;10:98.

-39 Paez JG, Janne PA, Lee JC, Tracy S, Greulich H, Gabriel S, Herman P, Kaye FJ, Lindeman N, Boggon TJ, Naoki K, Sasaki H, Fujii Y, Eck MJ, Sellers WR, Johnson BE, Meyerson M: EGFR mutations in lung cancer: correlation with clinical response to gefitinib therapy. Science 2004;304:1497-1500.

-40 Sequist LV, Martins RG, Spigel D, Grunberg SM, Spira A, Janne PA, Joshi VA, McCollum D, Evans TL, Muzikansky A, Kuhlmann GL, Han M, Goldberg JS, Settleman J, Iafrate AJ, Engelman JA, Haber DA, Johnson BE, Lynch TJ: First-line gefitinib in patients with advanced non-small-cell lung cancer harboring somatic EGFR mutations. J Clin Oncol 2008;26:2442-2449.

-41 Zhao JJ, Lin J, Zhu D, Wang X, Brooks D, Chen M, Chu ZB, Takada K, Ciccarelli B, Admin S, Tao J, Tai YT, Treon S, Pinkus G, Kuo WP, Hideshima T, Bouxsein M, Munshi N, Anderson K, Carrasco R: miR-30-5p functions as a tumor suppressor and novel therapeutic tool by targeting the oncogenic Wnt/beta-catenin/BCL9 pathway. Cancer Res 2014;74:1801-1813.

42 Yang X, Chen Y, Chen L: The Versatile Role of microRNA-30a in Human Cancer. Cell Physiol Biochem 2017;41:1616-1632.

43 Wang K, Jia Z, Zou J, Zhang A, Wang G, Hao J, Wang Y, Yang S, Pu P: Analysis of hsa-miR-30a-5p expression in human gliomas. Pathol Oncol Res 2013;19:405-411.

44 Liu J, Wu X, Liu H, Liang Y, Gao X, Cai Z, Wang W, Zhang H: Expression of microRNA-30a-5p in drug-resistant and drug-sensitive ovarian cancer cell lines. Oncol Lett 2016;12:2065-2070. 